\title{
Structure of stable binary neutron star merger remnants: A case study
}

\author{
W. Kastaun, R. Ciolfi, and B. Giacomazzo \\ Physics Department, University of Trento, via Sommarive 14, I-38123 Trento, Italy \\ and INFN-TIFPA, Trento Institute for Fundamental Physics and Applications, \\ via Sommarive 14, I-38123 Trento, Italy \\ (Received 7 July 2016; published 29 August 2016)
}

\begin{abstract}
In this work, we study the merger of two neutron stars with a gravitational mass of $1.4 M_{\odot}$ each, employing the Shen-Horowitz-Teige equation of state. This equation of state is a corner case, allowing the formation of a stable neutron star with the given total baryonic mass of $3.03 M_{\odot}$. We investigate in unprecedented detail the structure of the remnant, in particular the mass distribution, the thermal structure, and the rotation profile. We also compute fluid trajectories both inside the remnant and those relevant for the formation of the disk. We find a peanut-shaped fluid flow inside the remnant following a strong $m=2$ perturbation. Moreover, the flow is locally compressive, causing the appearance of dynamic hot spots. Further, we introduce new diagnostic measures that are easy to implement in numeric simulations and that allow one to quantify mass and compactness of merger remnants in a well-defined way. As in previous studies of supra- and hypermassive stars, we find a remnant with a slowly rotating core and an outer envelope rotating at nearly Keplerian velocity. We compute a Tolman-Oppenheimer-Volkoff star model which agrees well with that of the remnant in the core, while the latter possesses extensive outer layers rotating close to Kepler velocity. Finally, we extract the gravitational wave signal and discuss the detectability with modern observatories. This study has implications for the interpretation of gravitational wave detections from the postmerger phase and is relevant for short gamma-ray burst models.
\end{abstract}

DOI: 10.1103/PhysRevD.94.044060

\section{INTRODUCTION}

Binary neutron star (BNS) mergers are one of the most interesting astrophysical scenarios since their description requires both general relativity (GR) in the strong field regime as well as nuclear physics for matter above nuclear density, in particular the equation of state (EOS) and neutrino physics. BNS mergers are also promising candidates for multimessenger astronomy. First, they are among the most promising sources of gravitational waves for the detection with ground-based interferometers such as advanced LIGO [1] and Virgo [2]. Second, they are thought to be the energy source of short gamma ray bursts (SGRBs) [3], which are frequently observed among the most energetic explosions known in the universe. Furthermore, these events represent the most likely explanation for the abundance of heavy elements in the universe, complementary to supernova explosions $[4,5]$. The hot and neutron-rich ejecta from the merger process can indeed undergo rapid neutron capture nuclear reactions ( $r$-process reactions) which produce the heavy elements. Moreover, the radioactive decay of these elements heats up the material and leads to a so-called kilonova (or macronova) signal [6-8], emerging mostly in the optical band days/weeks after merger. Candidates for such a kilonova have already been found $[9,10]$. Finally, in addition to SGRBs and kilonova signals, BNS mergers may be accompanied by other electromagnetic counterparts, including late-time radio signals (e.g., [8]) and, if the remnant is not a black hole $(\mathrm{BH})$ but a long-lived metastable (or stable) NS [11], luminous signals powered by the remnant spin-down and peaking in the $\mathrm{x}$-ray band $[12-14]$ or at lower energies $[15,16]$.

The recent direct detection of two gravitational wave events caused by binary BH mergers $[17,18]$ has raised expectations for a detection of GWs from BNS mergers in the near future [19]. Such a detection might include a relatively strong signal from the postmerger phase. While the postmerger signal of binary $\mathrm{BH}$ mergers always consists of a short $\mathrm{BH}$ ringdown, for a BNS merger it can be much more complex. Moreover, this signal offers a unique opportunity to constrain the NS EOS [20-22].

The outcome of a BNS merger depends largely on the total mass of the system: while heavy systems promptly collapse to a $\mathrm{BH}$ during merger, lighter systems form a metastable or even stable NS. Merger remnants can be classified based on their mass. A hypermassive NS has a mass exceeding the maximum mass of a uniform rotating star, and a supramassive NS is below that mass but above the maximum mass for a nonrotating configuration. Those masses are also used to predict the lifetime of the merger remnant. A hypermassive neutron star (HMNS) collapses within tens of milliseconds, as soon as the degree of differential rotation becomes insufficient to stabilize it. Above a certain threshold mass, however, the merger results in a prompt collapse without a HMNS phase [23]. A supramassive neutron star (SMNS) can be supported by uniform rotation for much longer spin-down time scales, and eventually collapses when the rotational support 
becomes too small. In the early postmerger phase $(<100 \mathrm{~ms})$ the associated loss of angular momentum can be dominated by GW emission, while magnetic braking likely dominates on longer time scales.

The exact values of the masses dividing the above cases depend sensitively on the EOS. Hence, the presence of a postmerger signal could rule out all EOS for which the masses determined from the inspiral signal would lead to a prompt collapse. Moreover, the detection of a very long postmerger signal (although more difficult due to decaying amplitude) could even rule out EOS predicting a HMNS as the outcome, instead of a SMNS. In addition, the postmerger GW spectrum is typically dominated by a single peak around a few kilohertz, which also depends on the EOS. Measuring the corresponding frequency might allow for an additional constraint on the EOS [20-22].

In addition to the postmerger GW signal, there are additional reasons to consider a BNS merger leading to a long-lived remnant NS as a very important case. First, the recent discovery of single NSs with a mass of $\sim 2 M_{\odot}$ $[24,25]$ combined with the fact that the expected distribution of progenitor masses in BNS mergers is peaked around 1.3-1.4 $M_{\odot}$ [26] leads to the conclusion that forming a SMNS is a likely possibility, and in some cases it might be possible to form even a stable NS [11].

A second reason is that recent observations of SGRBs by Swift [27] revealed a large fraction of events accompanied by long-lasting (from minutes to hours) x-ray afterglows, which suggests the presence of a continuous injection of energy from a long-lived central engine (e.g., [28]). If SGRBs are associated with BNS mergers, this would suggest the presence of a long-lived (supramassive or stable) NS, instead of the commonly assumed BH surrounded by an accretion disk. The main difficulty of this socalled "magnetar model" is the fact that in this case the strong baryon pollution surrounding the merger site could choke the formation of a relativistic jet and thus prevent the SGRB itself. To overcome such limitations, an alternative "time-reversal" scenario [29] has been proposed, in which a SMNS survives for some time powering the long-lasting $\mathrm{x}$-ray emission and eventually collapses to a $\mathrm{BH}$ and launches the relativistic jet. Because of the optical depth of the surrounding environment, the spin-down-powered $\mathrm{x}$-ray emission is delayed and can still be observed after the SGRB (thus appearing as an "afterglow"). The main challenge to this scenario is the formation of a massive disk surrounding the $\mathrm{BH}$, when the latter is formed not shortly after merger, but minutes or hours later. Studying the rotation profile, the disk structure and the amount of ejecta surrounding the merger site in the case of a long-lived NS is therefore necessary in order to shed light on the different SGRB scenarios.

Models of differential rotation in the remnant NS often assume a rapidly rotating core, with the rotation profile described by the so-called $j$-const law [30]. In contrast, a recent study [31] demonstrated that HMNS or SMNS can indeed be produced with a slowly rotating core. In the case of HMNSs, the collapse can be significantly delayed because part of the mass forms an extensive outer bulge rotating close to Kepler velocity, thus centrifugally supported. Further studies [32] found similar behavior for more models, including an unequal mass system. The rotation profile is important for the oscillation frequencies of the core and hence the radiated GW signals. Also, the lifetime of a HMNS stabilized by a rapidly rotating core could differ strongly from a HMNS stabilized by a rapidly rotating outer bulge. This should be taken into account when attempting to deduce the EOS from the duration of a postmerger GW signal. Moreover, the differential rotation profile may also have an effect on magnetic field amplification and electromagnetic emission, as seen in previous studies employing the $j$-const law [33]. Therefore a careful investigation of the remnant properties also has important applications to electromagnetic counterparts of GWs from BNS mergers and, possibly, to SGRBs.

Beside rapid differential rotation and larger mass, another difference to isolated NS is that shock heating during merger requires the consideration of thermal effects. A common notion is that HMNS are also stabilized by thermal pressure. For nuclear physics EOS, however, the temperatures reached during merger do not increase the maximum mass of spherical or uniformly rotating NSs strongly, and, somewhat counterintuitively, can even decrease it $[34,35]$. However, the merger remnants are not heated homogeneously. It is possible that underdensities caused by "hot spots" in the rapidly rotating remnant contribute to the GW signal, with a frequency determined by the rotation rate at the location of the hot spot. Unless the hot spots occur near a maximum of the rotation rate, they would quickly turn into tightly wound spirals because of differential rotation, at which point the associated GW signal ceases. Because of this effect, both the exact nature of the fluid flow inside the remnant and the thermal structure, as well as their interplay, could become important for the interpretation of future GW detections. Note, however, that such contributions are likely smaller than the one from the nonaxisymmetric oscillation of the remnant. Further, this effect competes with other secondary features caused by nonlinear combination frequencies between the radial and the main nonaxisymmetric oscillation [36] or side peaks caused by a strong frequency modulation of the main peak [31], again caused by radial oscillations. Even with a good signal to noise ratio for the postmerger phase, deriving information from secondary features of the postmerger spectrum will require a good theoretical understanding of all those effects.

In this work, we will focus on a binary with typical mass but an EOS that allows the formation of a stable NS. This case is generally considered rather unlikely, and for this reason it has not been investigated in detail. We note, 
however, that most of the results that will be presented in this study are also indicative for the SMNS case, which is instead a likely outcome of a BNS merger. We will investigate the structure of the fluid flow and thermal structure with regard to possible contributions to the postmerger GW signal. We will also investigate the rotation profile and mass distribution, and then we will take a closer look at the formation of the disk surrounding the remnant. Finally, we provide estimates for the mass ejection.

Throughout this paper, we use units $G=c=1$ unless noted otherwise. We define baryonic mass $M_{b}=N_{b} m_{b}$ and rest mass density $\rho=n_{b} m_{b}$, where $N_{b}$ and $n_{b}$ are baryon number and baryon number density in the fluids rest frame, respectively, and $m_{b}$ is a formal mass constant $m_{b}=931.494 \mathrm{MeV}$.

\section{SETUP}

\section{A. Models}

In this study, we consider an irrotational binary neutron star system consisting of two stars with a gravitational mass (in isolation) of $1.4 M_{\odot}$ each. We use the Shen-HorowitzTeige (SHT) EOS [37,38], which is based on a relativistic mean field theory method and a modified NL3 set of interaction parameters. The initial model is at zero temperature and in $\beta$ equilibrium.

The total baryonic mass of the binary is $3.03 M_{\odot}$. For such a high mass, the outcome of the merger would be a HMNS or a prompt collapse for most EOS. The SHT EOS, however, features an unusually large maximum mass of $3.38 M_{\odot}$ for Tolman-Oppenheimer-Volkoff (TOV) stars. The maximum mass for uniformly rotating stars is $3.97 M_{\odot}$. Therefore, we expect that a stable NS is formed after merger.

The initial data were computed using the LORENE code [39]. Note that because the quasicircular approximation employed in the formalism ignores the radial velocity component of the inspiral, the resulting models always show a small eccentricity when evolved. We did not attempt to correct for this, as done, e.g., in [40], since the eccentricity is mainly a problem when studying tidal effects during the inspiral. The initial proper separation of our model is $57.6 \mathrm{~km}$, which corresponds to 4 orbits until merger.

\section{B. Numerical methods}

To evolve the general relativistic hydrodynamic equations, we use the WhiskyThermal code described in $[35,41]$. It is based on a finite-volume high resolution shock capturing scheme that uses the Harten-Lax-van Leer-Einfeldt approximate Riemann solver and the piecewise parabolic method for reconstructing values at the cell interfaces. We employ a tabulated equation of state including thermal and composition effects in terms of the electron fraction. We do not consider magnetic fields and neutrino radiation, and the electron fraction is passively advected along the fluid.
Our code features a robust method (see [35]) for the conversion from evolved to primitive variables that also includes stringent checks of the evolved variables, with a clear error policy, allowing for adjustments of harmless numerical errors such as temperatures falling slightly below zero when evolving cold initial data, but aborting the run in case of severe errors. The error policy used here is the same as described in [35].

For this work, we also included the option to enforce adiabatic evolution at zero temperature. The motivation is to avoid artificial heating at the NS surfaces during the inspiral, which is a cumulative effect. To enforce zerotemperature evolution, we evolve in the same way as before, including the energy, but recompute the conserved energy after each evolution substep from conserved density and momentum, assuming zero temperature. For this, we modified our conservative to primitive scheme by replacing the specific energy computed from the evolved variables by the value obtained from the EOS evaluated at zero temperature (more precisely, at the lowest tabulated value, which is low enough for our purpose). This way, the evolved conserved energy is not used at all. Around a ms before the stars touch, we activate the full thermal evolution.

For the evolution of the spacetime, we use the McLachlan code [42], which is part of the Einstein Toolkit [43]. This code implements two formulations of the evolution equation. Instead of the popular Baumgarte-Shibata-ShapiroNakamura formulation [44-46], we chose the newer conformal and spatially covariant CCZ4 evolution scheme described in $[41,47]$, which has constraint damping capabilities. The gauge conditions used during evolution are the $1+\log$-slicing condition [48] for the lapse function and the hyperbolic $\Gamma$-driver condition [49] for the shift vector. At the outer boundary, we use the Sommerfeld radiation boundary condition.

All codes are integrated into the Cactus Computational Toolkit infrastructure. The time evolution is coupled using the method of lines, in particular we use a fourth order Runge-Kutta time integrator. Further, we make use of Berger-Oliger moving-box mesh refinement provided by the Carpet code [50]. In detail, we use six refinement levels. The two finest ones follow the stars during inspiral, and are replaced by fixed levels centered around the origin shortly before merger. The finest grid spacing is $295 \mathrm{~m}$. The outer boundary is located at $945 \mathrm{~km}$, and the finest level after merger covers a radius of $30 \mathrm{~km}$. Finally, we use reflection symmetry across the orbital plane. For tests of the code, we refer the reader to $[35,41]$.

\section{Analysis tools}

Since we are interested in the perturbation of the merger remnant, we need to be able to distinguish between actual deformations and mere gauge effects. In particular the spatial coordinates in GR simulations can be problematic for the analysis of deformations, since the gauge conditions 
typically used are mainly designed to prevent coordinate pathologies, not to seek symmetries. Instead of changing the gauge conditions during the simulation, we transform to a more suitable coordinate system during the postprocessing stage. The new coordinates are obtained following a well-defined prescription we described in detail in [31].

Those coordinates are restricted to the orbital plane (and require reflection symmetry). The main advantages are that the radial coordinate is parametrized by proper length, that the radial coordinates are on average orthogonal to the $\phi$-coordinates, preventing spiral deformations, and, most important, that the $\phi$-coordinate base vector becomes a Killing vector for an axisymmetric spacetime.

To measure deformations of the remnant, we use a decomposition in $\phi$-harmonic components as described in $[31]$

$$
P_{m}^{q}=\int_{0}^{R} \int_{0}^{2 \pi} q(r, \phi) e^{i m \phi} \mathrm{d} A,
$$

where $q$ stands for an arbitrary scalar variable, $\mathrm{d} A$ is the proper area element in the new coordinates $(r, \phi)$, and $R$ is a suitable cutoff radius larger than the remnant. We are mostly interested in $P^{\rho}$, for which we use $R=40 \mathrm{~km}$, large enough to fully contain the remnant.

Besides oscillations of the remnant, we also want to measure its radial structure. This is, however, complicated by the fact that merger remnants are rapidly and differentially rotating objects, which typically also exhibit strong nonaxisymmetric oscillations excited during the merger. Together with the ambiguity of defining radii in GR, it becomes nontrivial to define, e.g., the density profile in the same way as for a spherically symmetric static star. To solve this issue we consider, for a given time slice, surfaces of constant baryon mass density (baryon number density), measured with respect to the fluid rest frame. Those only depend on the chosen foliation of spacetime, but not on any spatial coordinate system. For each density isosurface, we can measure the total enclosed proper 3-volume $V$, where the enclosed region is defined as the region occupied by matter with higher densities. In the same way, we define the total enclosed baryon mass $M_{b}$. This way, we have established a mass-versus-volume relation that is a suitable replacement for density-versus-radius relations used to describe spherical stars. For convenience, we also define a volumetric radius $R_{V}$ as $V=\frac{4}{3} \pi R_{V}^{3}$.

Note that although this measure is mainly intended for objects of spherical topology, it is well defined for any density distribution. Examples with the topology of a torus are given by the disk around a merger remnant, as well as the highdensity regions of some hypermassive NS models featuring a ring-shaped density maximum. For such cases, the volumetric radius cannot be interpreted as a measure of the object size, but the mass-volume relations can still be used to make unambiguous comparisons between such distributions.
Another important measure of spherical stars is the compactness $M / R$, usually defined in terms of gravitational mass and circumferential radius. Since both are hard to define unambiguously for merger remnants in GR, we use the baryon mass and the volumetric radius instead to define the compactness $C_{V}=M_{b} / R_{V}$ of a given isodensity surface. Defining the compactness of the remnant, we encounter another obstacle given by the fact that instead of a NS surface there is a smooth transition to the surrounding disk and other merger debris. To circumvent this, we use the observation that our new compactness measure $C_{V}$ has a clear maximum. We define the "bulk" as the region enclosed by the isodensity surface with the maximum compactness, and the enclosed baryon mass and proper volume as bulk mass $M_{\mathrm{blk}}$ and bulk volume $V_{\mathrm{blk}}$. Note that $C_{V}$ is designed for use with isolated stars and merger remnants, where the bulk will most likely always be a moderately deformed sphere. Although still well defined when applied to a torus, for example, it could not be interpreted any more as mass within some average radius.

In order to motivate the tentative name choice bulk, we now derive some expressions for the density and density gradient on the bulk surface. For this discussion, we assume that the isodensity surfaces can be used as a smooth foliation of space and express the integrals for enclosed volume and mass as

$$
\begin{gathered}
V\left(\rho^{\prime}\right)=\int_{\rho^{\prime}}^{\infty} \int_{A(\rho)} \mathrm{d} V, \\
M_{b}\left(\rho^{\prime}\right)=\int_{\rho^{\prime}}^{\infty} \int_{A(\rho)} W \rho \mathrm{d} V,
\end{gathered}
$$

where $\mathrm{d} V$ is the proper 3-volume element, $A(\rho)$ the isodensity surface of rest-mass density $\rho$, and $W$ the fluid Lorentz factor. Decomposing the volume element as $\mathrm{d} V=N \mathrm{~d} A \mathrm{~d} \rho$, where $\mathrm{d} A$ is the proper area element on $A(\rho)$, and $N$ is a scalar function (analogous to the lapse function of space-time foliations), we find

$$
\frac{d M_{b}}{d V}=\rho \bar{W}(\rho), \quad \bar{W}(\rho)=\frac{\int_{A(\rho)} W N \mathrm{~d} A}{\int_{A(\rho)} N \mathrm{~d} A},
$$

and, together with the definition of $C_{V}$,

$$
\frac{d C_{V}}{d V}=V^{-\frac{1}{3}}\left(\rho \bar{W}(\rho)-\frac{M_{b}}{3 V}\right)
$$

Thus, a surface of maximum compactness has to satisfy $3 \bar{W}\left(\rho_{\mathrm{blk}}\right) \rho_{\mathrm{blk}}=M_{\mathrm{blk}} / V_{\mathrm{blk}}$. In other words, the density of the bulk surface (times its average Lorentz factor $\bar{W}$ ) is one third of the average interior density $M_{b} / V$.

Evaluating the second derivative of $C_{V}$ on the maximum compactness surface, we find 


$$
0>\frac{d^{2} C_{V}}{d V^{2}}=V^{-\frac{4}{3}}\left(\frac{2}{3} \bar{W} \rho+V \frac{d}{d V}(\bar{W} \rho)\right) .
$$

Inserting the definition of $R_{V}$, we obtain

$$
\frac{d \ln (\bar{W} \rho)}{d \ln R_{V}}<-2
$$

Hence, $\bar{W} \rho$ falls off faster than $R_{V}^{-2}$ near the bulk surface. When applied to our merger simulations, the definition of the bulk excludes the outermost low-density layers of the remnant as well as the disk. We note that the bulk properties are influenced by rapid rotation and nonradial oscillations only via changes of average density, not by the deformation of the shape. In the limit of a constant density star and flat space, the bulk properties are even completely independent of its shape.

Besides baryon number and proper volume, one can also compute the total entropy $S_{\text {blk }}$ as well as the number of electrons inside the bulk, defining the bulk average specific entropy $\bar{s}_{\text {blk }}=S_{\text {blk }} / M_{\text {blk }}$ and bulk average electron fraction. The former will be used in this work as a measure of heating during merger, and the latter might be useful in simulations involving neutrino cooling.

Of course, our new measures can also be applied to TOV solutions for spherical NSs. Compared to the surface radius, the bulk volume has the advantage that it is insensitive to the low density part of the star, and might thus be more useful for any computation that relates the dynamics of the whole star to the compactness, e.g., empirical estimates for BNS mergers. The bulk compactness might also be a candidate for universal relations of NS oscillation frequencies, replacing, e.g., the effective compactness defined from the moment of inertia (compare [51]).

The above measures are surprisingly easy to implement in a numerical simulation. We simply collect the proper volume and baryon mass of all grid cells into a onedimensional histogram where the bins are given by the logarithm of the rest mass density. The only complication is to make sure that points covered by several refinement levels are only counted once. The required masks are the same required for standard reduction operations, such as the average, and are therefore readily available. To get, e.g., the total mass inside a given isodensity surface, we then simply compute the reverse cumulative histogram to obtain the total mass of all cells with densities higher than the given one. Extracting the bulk then only requires a simple one-dimensional maximum search. From the histograms, one can also extract the isodensity surface which contains a given total baryon mass. This will be used to visualize the mass distribution.

In order to better understand the evolution during and after merger, we will study the trajectories of fluid elements in addition to the above measures. The usual method to obtain fluid trajectories is to evolve tracer particles during the simulation. The drawback of this approach is that the starting positions have to be chosen before the simulation, and it is difficult to obtain a good coverage of the computational domain at later stages. This is because the matter in the disk surrounding the remnant is the result of an expanding fluid flow, resulting in a tracer density which is much smaller than the original one. Using a large number of tracers is inefficient, and guessing where to increase the initial tracer density is error prone.

To circumvent those problems, we evolve the fluid trajectories backward in time in a postprocessing step. As starting positions, we simply use a regular grid near the end of the simulation (or at whatever time we wish a homogeneous coverage). This approach has the drawback that the data need to be saved with a high enough sampling rate to integrate the fluid trajectories with reasonable accuracy. We therefore restrict ourselves to the equatorial plane, which also has the advantage that we can use the well-defined coordinate system described above. In addition, we integrate the trajectories in polar coordinates which are better suited for following the rapid rotation of the remnant than Cartesian coordinates. Note also that when integrating backwards in time, the problematic expanding fluid flow becomes converging instead; thus an error in the integration is diminished over time.

Last but not least, we need to determine the amount of matter ejected to infinity during the merger. As described in [31], this is a nontrivial problem mainly due to the use of a constant-density artificial atmosphere in our code and because of the requirement to predict if a fluid element will eventually escape to infinity. We adopt the same tools described in previous works $[31,32]$. In short, we use the assumption of geodesic motion as predictor for unbound matter and then compute the flux of unbound matter through spherical surfaces of various radii as well as the volume integral over the unbound mass density. Further, we collect the unbound mass in bins of coordinate radius at regular time intervals to get a spacetime diagram of the mass ejection.

\section{RESULTS}

A visual overview of the evolution is given in Fig. 1, showing spacetime diagrams where two dimensions correspond to the equatorial plane and the third to time. The top panel depicts the evolution of the NS core(s). To obtain this figure, we use the histograms introduced in Sec. II C to compute for each time the function mapping a given density to the total mass of all matter which has higher densities. We then map the density in space and time to the fraction of this mass to the total mass. The plot shows the isosurface that contains one quarter of the mass. To reduce gauge effects, we use the special coordinates defined in Sec. II C for the orbital plane. 


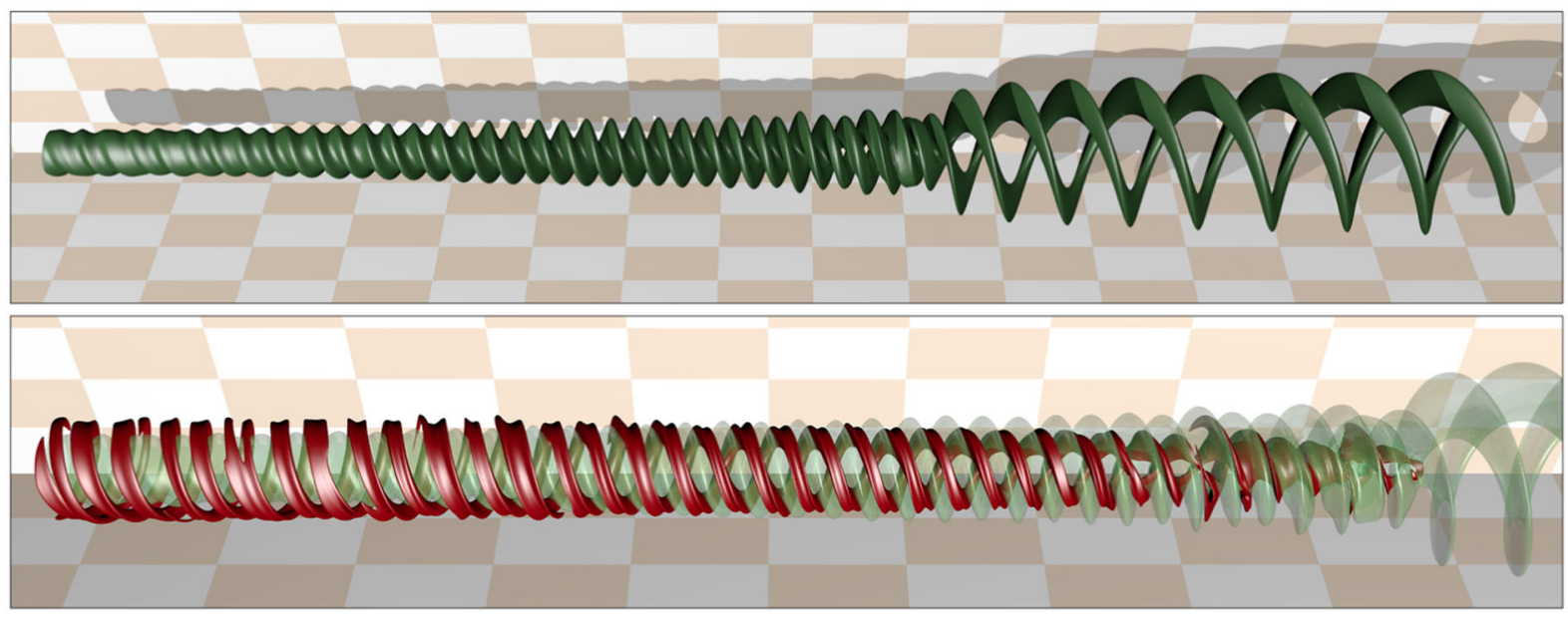

FIG. 1. Spacetime diagram showing the world tubes of different isocontours in the orbital plane. The time coordinate runs from right to left. Top panel: isodensity contour containing (at each time) $25 \%$ of the total baryon mass, showing the evolution of the core(s). Bottom panel: the red surface is the contour of constant entropy density chosen to highlight the evolution of the hot spots. For comparison, the surface in the top panel is shown again, but rendered transparent. The size of the tiles in the background is $2 \mathrm{~ms} \times 20 \mathrm{~km}$, and the lower panel is zoomed to show the postmerger phase.

As one can see, the system completes 4 orbits before merging. At merger, the system undergoes one "bounce," after which it briefly consists of two separate cores in a common envelope. Around $4 \mathrm{~ms}$ after merger, a single strongly deformed remnant has formed. Initially the deformation is dominated by the $m=2$-component. After $15 \mathrm{~ms}$, its amplitude is markedly decreased, and the influence of other components becomes visible. This will be discussed in detail in Sec. III B.

To visualize the thermal evolution, we show the world tube of the isocontours of entropy density in the equatorial plane in the lower panel of Fig. 1. We use the entropy density instead of temperature in order to focus on the remnant instead of the surrounding matter. A prominent feature visible in the figure is a two-armed helical structure in the postmerger phase, corresponding to two entropy concentrations in circular motion. These hot spots will be discussed in more detail in Sec. III B. Also note that during the double core phase, the entropy is concentrated around the origin. This is exactly what is expected from shock heating during merger. In the subsequent evolution, the entropy density pattern apparently changes very quickly.

The three-dimensional structure of the entropy distribution in the different evolution phases is shown in Fig. 2. The first snapshot shows the shock-heated region produced when the stars touch, featuring three different parts. Apparently, the "elephant ears" are produced because the stars touch off-center in two points, while the middle part is heated when the stars come in full contact. The second snapshot shows the fully developed shock-heated region which separates the aforementioned double cores. This region is quickly ripped into different parts by the complex fluid flow, as shown in the next snapshot. Shortly thereafter, the entropy is concentrated in the two hot spots discussed above. Those entropy concentrations exhibit a saddle shape shown in the lower left panel. This pattern stays stable for several milliseconds. Afterwards, the system becomes gradually more axisymmetric until it assumes a ring-shaped configuration shown in the lower right panel. The snapshots also show an isodensity surface containing $85 \%$ of the mass. This surface is strongly deformed up to the end of the simulation. In particular it exhibits a feature strongly resembling two wave crests, one of which is visible in the lower left part of the panel. By comparison with two-dimensional cuts, we found that those wave crests are the origin of two spiral waves that heat up the surrounding disk.

The evolution of the bulk mass defined in Sec. II C is shown in Fig. 3. After merger, it smoothly decreases from the value for the two isolated stars by $\approx 0.5 M_{\odot}$. This is due to the fact that a significant fraction of the material is spread out in a rapidly rotating outer envelope and the disk. Figure 3 also shows the total mass of matter with densities higher than the one at the origin. This measure is useful to quantify the double-core phase: the mass in the separate cores defined in this way is around 1.5-2 $M_{\odot}$, and the length of the double core phase is $\approx 2 \mathrm{~ms}$.

To quantify the compactness of the remnant, we use the bulk compactness defined in Sec. II C, which is shown in Fig. 4. For comparison, we also computed at each time the same measure for a single (cold) TOV star with the same bulk mass as the evolved system, as well as for a configuration of two isolated cold TOV stars. Note the bulk compactness of each single star alone is (by definition) lower by a factor of $2^{\frac{2}{3}}$. We find that the bulk compactness is very close to the one for two isolated stars right until the merger. Apart from some oscillations, it starts deviating significantly only after the double core phase. Not 

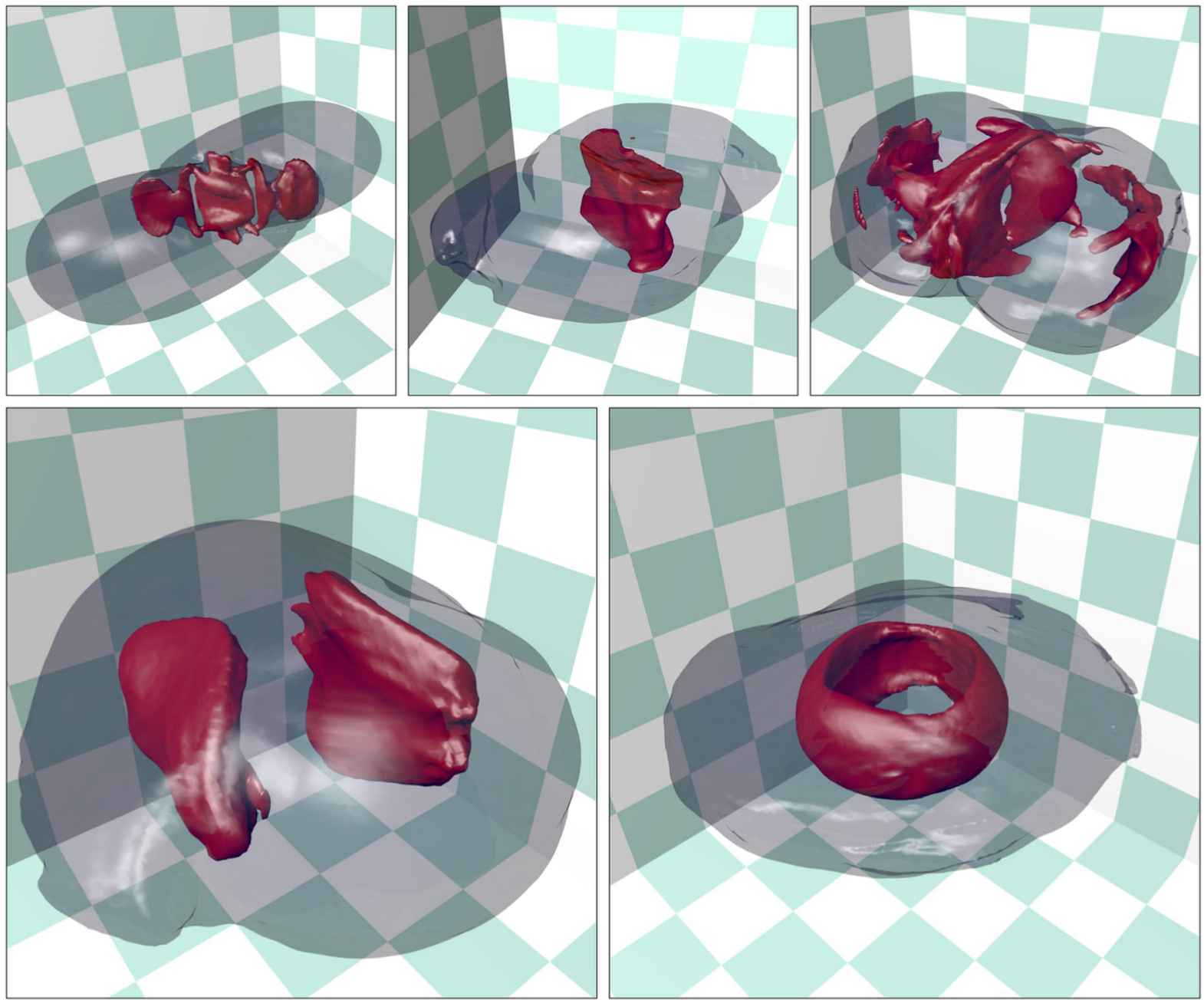

FIG. 2. Snapshots of entropy distribution at times (from top-left to bottom-right) $0.1,1.8,3.5,8.5$, and 18.3 ms after merger. The red surface is the isosurface of entropy density which contains $85 \%$ of the entropy. The transparent surface depicts the isosurface of mass density which contains a mass of $2.7 M_{\odot}$. The size of the tiles in the background is $10 \mathrm{~km}$.

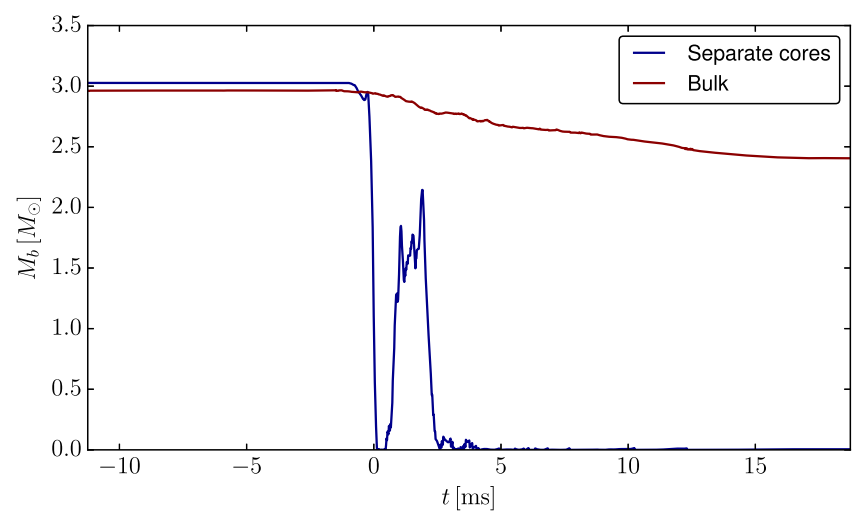

FIG. 3. Evolution of baryonic mass contained in the bulk and separate cores. The latter is defined as the mass of all matter with density higher than the one at the origin.

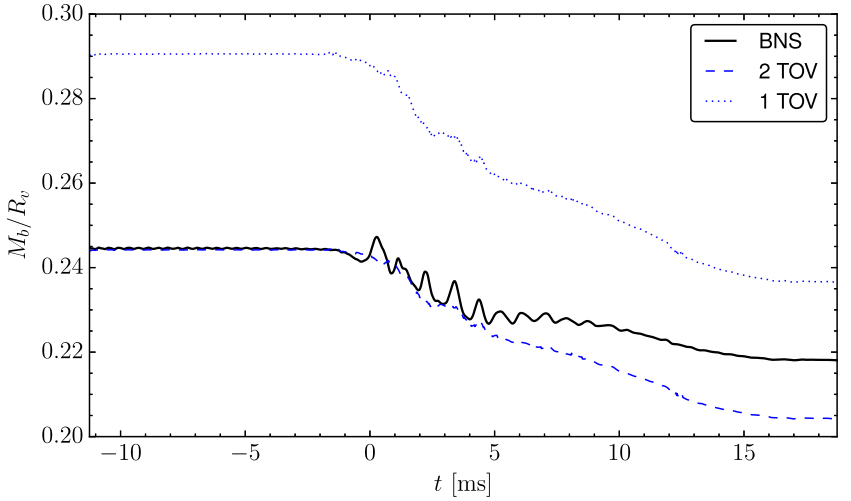

FIG. 4. Evolution of bulk compactness $M_{b} / R_{V}$ (solid line), where $R_{V}=((3 /(4 \pi)) V)^{1 / 3}$ is the volumetric radius of the bulk (see main text), $V$ its proper volume, and $M_{b}$ its baryon mass. For comparison, we show the same measure for a configuration with the same bulk mass, but consisting either of a single TOV star (dotted line) or of 2 TOV stars (dashed line). The EOS for the latter is the same as for the initial data. 
surprisingly, the bulk of the rotating remnant is less compact than a TOV star with the same mass. We will investigate the mass distribution in more detail in Sec. III A.

Next, we investigate the production of entropy during and after merger. For this, we first divide the system into bulk and everything else. We then compute a specific bulk entropy from the total entropy in the bulk divided by the baryon mass of the bulk. In the same fashion, we compute the specific entropy of the rest of the system, which we identify with the disk (once the disk has formed). The result is shown in Fig. 5. The average specific entropy of the bulk reaches $\approx 1 k_{B}$ per baryon around $5 \mathrm{~ms}$ after merger and then stays almost constant. This is consistent with the observation that no shock waves occur inside the bulk after this time. The disk, on the other hand, is continuously heated by shock waves originating from the strongly deformed rotating outer envelope of the remnant. Correspondingly, the specific entropy of the disk keeps increasing until the end of the simulation, albeit at a slowing rate. After $18 \mathrm{~ms}$, it reaches $5 k_{B}$ per baryon.

On a technical side note, we remark that the specific entropy of the bulk exterior jumps abruptly to $0.5 k_{B}$ shortly before merger when we switch from adiabatic evolution during inspiral to fully thermal evolution. At this point, there is no disk and the exterior of the bulk consists of the NS surface, which is notorious for numerical errors in hydrodynamic codes. The jump in the total entropy is insignificant compared to the final value. However, the heating at the NS surface due to numerical errors might have been more significant if it had been allowed to accumulate during the whole inspiral. Since we observed no adverse side effects from enforcing adiabatic evolution during inspiral, we recommend it for similar simulations.

Using histograms summing the entropy and baryonic mass of numerical grid cells in (logarithmic) bins of rest

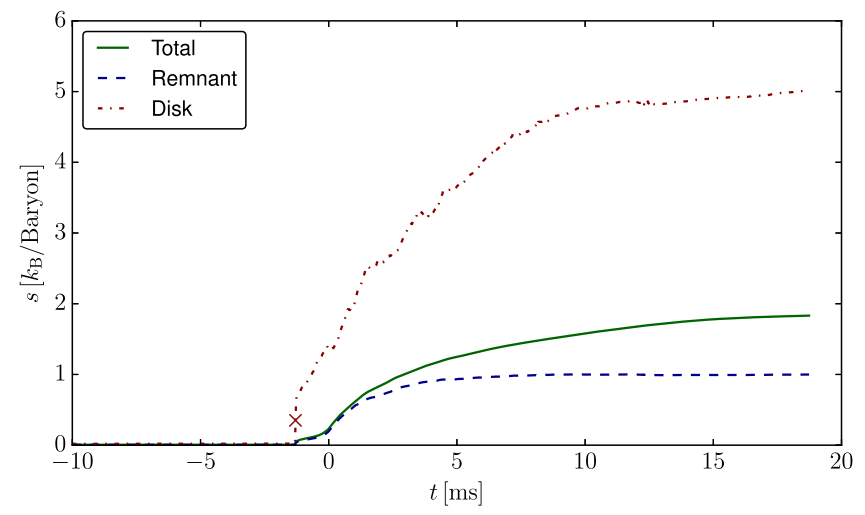

FIG. 5. Evolution of average specific entropy for matter inside and outside the bulk mass, labeled tentatively as remnant and disk. The average specific entropy is defined as the ratio of total entropy and total baryon number. The cross marks the activation of the thermal evolution. mass density, we obtain the average specific entropy for matter at given density, defined as the entropy in the corresponding bin divided by the baryonic mass. The result is shown in Fig. 6. We note that we do not consider neutrino radiation, which would likely reduce the disk temperature. Also note that the temperature inside the remnant is not just a function of density, as will be discussed in Sec. III B.

\section{A. Remnant rotation and mass profiles}

We now discuss the density profile of the remnant near the end of the simulation, after it had time to settle down. As discussed in Sec. II C, it is difficult to specify profiles in terms of radii unambiguously in GR in the absence of spherical symmetry. Therefore, we compute the total baryonic mass and the proper volume inside the isosurfaces of mass density, obtaining a mass-inside-volume relation that is completely independent of the spatial coordinates. Fig. 7 shows the relation for the remnant. For comparison, we also show this relation for one of the initial NSs at isolation.

Clearly, there is no well defined boundary between remnant and disk. To make quantitative statements about the remnant, we will use the definition of the bulk as the isodensity surface with the maximum compactness (in the sense defined in Sec. IIC). The position of the remnant bulk is marked in the figure as well, and its main properties are listed in Table I. Figure 7 also contains the relation of bulk mass versus bulk volume for a sequence of TOV stars with the same EOS as the initial data. Not surprising, the remnant bulk occupies more volume than a TOV star with the same bulk mass.

Note that the curve corresponding to the TOV sequence intersects the mass-inside-volume relation of the remnant. The mass-inside-volume profile for the TOV star at the intersection is also shown in Fig. 7. It is noteworthy that the profile of this TOV star and the remnant agree very well inside the bulk of the TOV star. In the following we call the latter the "TOV core equivalent." Its properties are given in

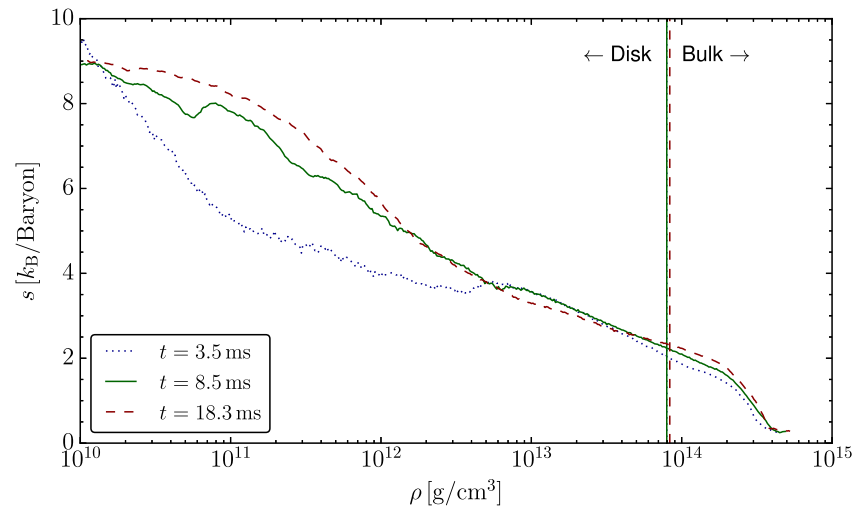

FIG. 6. Average specific entropy of matter versus rest mass density. The time in the labels is the time after merger. The vertical lines mark the density defining the bulk. 


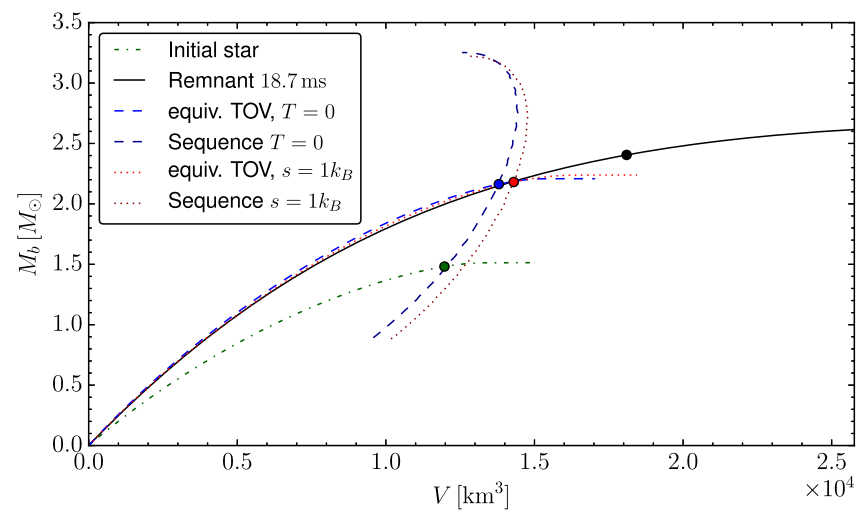

FIG. 7. Mass distribution of the remnant $18.7 \mathrm{~ms}$ after merger. Plotted is the baryonic mass versus the proper volume contained in surfaces of constant mass density. For comparison, we also show the same profile for one of the initial stars in isolation, and for the TOV core equivalents (see main text) with zero temperature as well as constant specific entropy of $1 k_{B}$. The symbols mark, from left to right, the bulk (see main text) of initial star, cold and hot core equivalent, and remnant. Further, we show the bulk mass versus the bulk volume for the two sequences of TOV stars with $T=0$ and $s=1 k_{B}$.

Table I, which also lists the central densities. Note the central density of the remnant and the TOV core equivalent agree within $3 \%$. We conclude that the average density of the core is not affected significantly by rotation and thermal effects. Note, however, that our measure is rather insensitive to nonradial deformations and cannot be used to draw conclusions on the oblateness or nonradial oscillation amplitude of the core.

To estimate the influence of thermal effects on the core, we also computed the TOV sequence and TOV core equivalent assuming beta equilibrium and a constant specific entropy of $1 k_{B}$, which is the average value for the bulk of the remnant (cf. Fig. 5). The bulk properties of the hot TOV core equivalents are listed in Table I. The hot TOV core equivalent indeed fits the remnant core better. However, as shown in Fig. 7, the differences to the cold star are rather small. The thermal pressure is therefore

TABLE I. Bulk properties of remnant at $18.7 \mathrm{~ms}$ after merger, of one of the stars at the beginning of the simulation, and of the remnant TOV core equivalent. The latter is computed both for zero temperature (cold TOV) and for constant specific entropy of $1 k_{B}$ (hot TOV). $M_{\mathrm{blk}}$ is the baryonic mass of the bulk, $V_{\mathrm{blk}}$ its proper volume, $\bar{s}_{\mathrm{blk}}=S_{\mathrm{blk}} / M_{\mathrm{blk}}$ its average specific entropy, and $S_{\text {blk }}$ its total entropy. $\rho_{c}$ is the central density.

\begin{tabular}{lcccc}
\hline \hline & $M_{\text {blk }}\left[M_{\odot}\right]$ & $V_{\text {blk }}\left[10^{4} \mathrm{~km}^{3}\right]$ & $\bar{s}_{\text {blk }}\left[k_{B}\right]$ & $\rho_{c}\left[10^{14} \mathrm{~g} / \mathrm{cm}^{3}\right]$ \\
\hline Remnant & 2.405 & 1.81 & 1.0 & 5.30 \\
Cold TOV & 2.163 & 1.38 & 0 & 5.47 \\
Hot TOV & 2.181 & 1.43 & 1 & 5.40 \\
Initial NS & 1.481 & 1.20 & 0 & 4.30 \\
\hline \hline
\end{tabular}

unimportant for the overall remnant core density in the case under consideration. As we will see in the next section, it is more relevant for nonaxisymmetric deformations of the core. Also the outer layers are typically affected more strongly by thermal effects; e.g., the surface radius of the hot TOV core equivalent is considerably larger compared to the cold one.

We now investigate the rotation profile of the remnant. To reduce gauge effects, we use the coordinate system in the orbital plane described in Sec. II C to compute the average angular velocity (as seen from infinity) along coordinate circles. The angular velocity as a function of time and circumferential radius is shown in Fig. 8. As one can see, directly after merger the average rotation rate in the core is highest. Note, however, that during this phase the system is not even approximately axisymmetric or stationary. In any case, the fluid flow quickly rearranges itself, and $4 \mathrm{~ms}$ after merger the core rotates slower than the parts at larger radii.

During the rearrangement phase, the development of a Kelvin-Helmholtz instability is expected. We note that the numerical resolution is too low to resolve it in full. This might have an effect on the final rotation profile, which is, however, difficult to quantify without very high resolution simulations.

The rotation profile after the remnant has settled down is shown in Fig. 9. To minimize the influence of remaining oscillations, we averaged in time between $13-15 \mathrm{~ms}$. We find that the maximum angular velocity is around $6 \mathrm{rad} / \mathrm{ms}$ and occurs at radii in the range $15-20 \mathrm{~km}$. In order to compare the rotation rate to the Kepler velocity, we also averaged the spacetime in time and $\phi$ direction, and we computed the angular velocities of test masses in corotating circular orbits, as described in [31]. The result is shown in Fig. 9, as well as the contribution of frame dragging to the

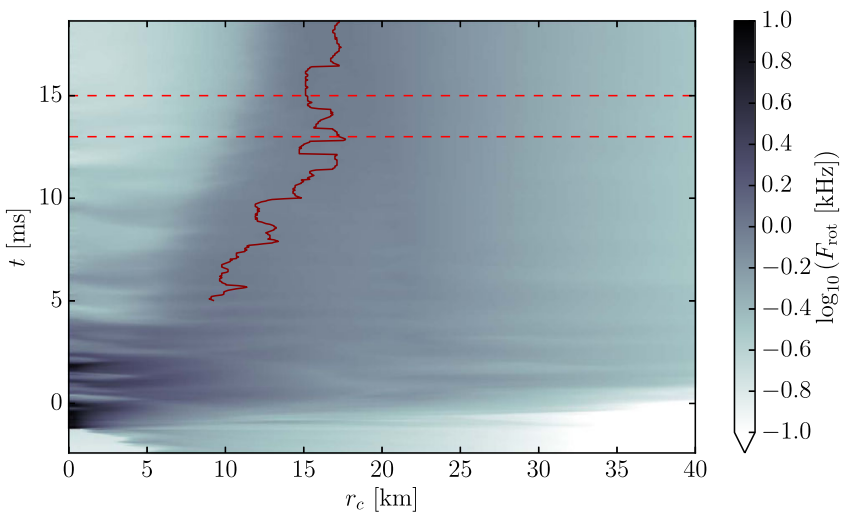

FIG. 8. Evolution of $\phi$-averaged angular velocity in the equatorial plane. $r_{c}$ is the circumferential radius and $t$ the coordinate time relative to the merger time. The location of the maximum after the merger phase is marked by the solid curve. The dashed horizontal lines mark the boundaries of the timeaverage shown in Fig. 9. 


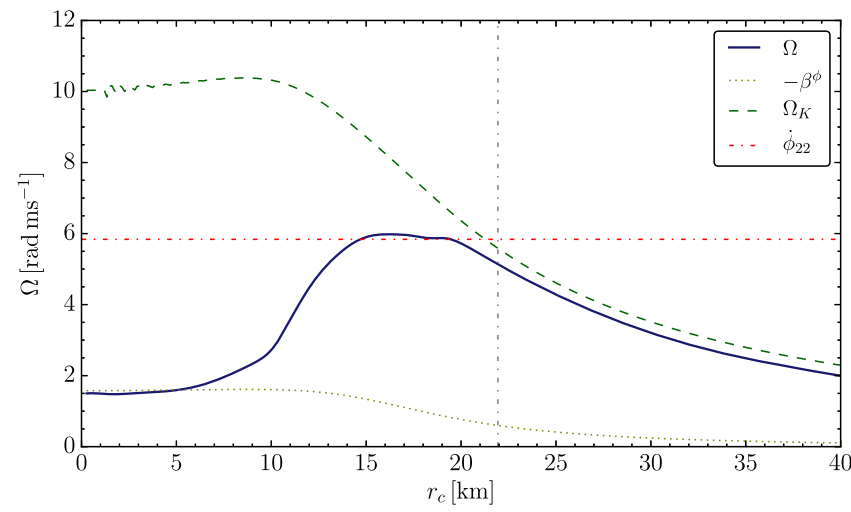

FIG. 9. Rotation profile of the remnant $14 \mathrm{~ms}$ after merger. Shown is the angular velocity $\Omega$ in the equatorial plane averaged in the $\phi$ direction as well as in time over a $2 \mathrm{~ms}$ window (13-15 ms). For comparison, the green dashed line is the orbital angular velocity $\Omega_{K}$ of test particles in corotating circular orbits. The dotted grey line is the frame-dragging contribution. The horizontal line is the pattern angular velocity $\dot{\Phi}_{22}=\dot{\phi} / 2$ of the $l=m=2$ component of the gravitational signal at the same (retarded) time. The vertical line marks the radius where the density falls below $5 \%$ of the maximum one.

angular velocity. We find that the core rotates indeed much slower than the local Kepler velocity, and is basically nonrotating in the local inertial frame. Further, we find that the outer layers approach the Kepler velocity.

Note that similar rotation profiles have already been observed in [31,32] for other binary models with different EOS and masses resulting in hyper- and supramassive remnants. Figure 9 also exhibits a feature not yet explained: the maximum rotation rate is very close to the angular velocity of the $l=m=2$ component of the GW pattern. This too has been observed in $[31,32]$ and thus seems more than a coincidence.

\section{B. Remnant deformation and thermal structure}

In this section, we will describe the evolution of the nonaxisymmetric density perturbation of the remnant, the hot spots, the fluid flow, and their relation to each other.

The simplest quantitative measure for the deformation of the remnant is the decomposition of the density in the equatorial plane into $\phi$-harmonic components, as described in Sec. II C. The moments $P_{m}^{\rho}$ up to $m=4$ are shown in Fig. 10 as a function of time. Clearly, the merger excites $m=2$ and $m=4$ deformations (the latter probably being a nonlinear overtone of the former). Since we study an equal mass system, no odd components are excited at merger. During the evolution, however, we observe a growing $m=3$ and $m=1$ contribution which becomes comparable to the $m=2$ component at the end of the simulation. The mechanism of this instability is not explained yet. The occurrence of $m=3$ modes for other equal mass systems has already been reported in [52].

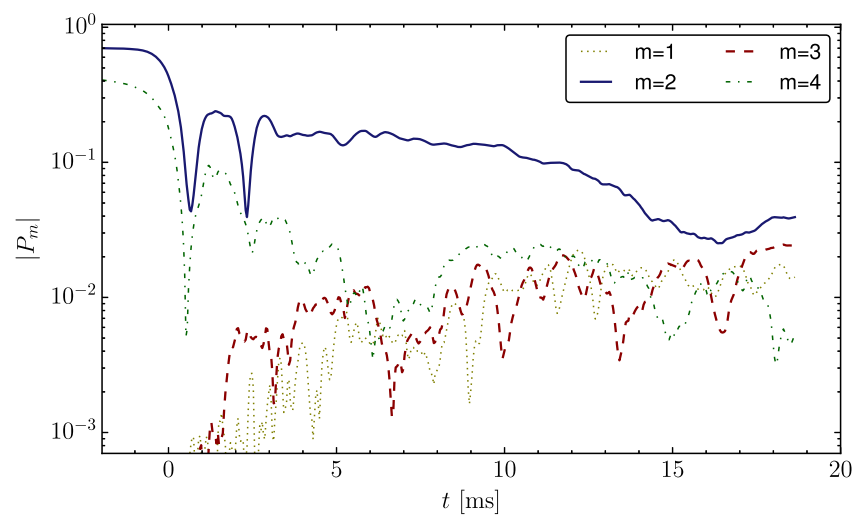

FIG. 10. Evolution of mass density moments in the equatorial plane.

We now turn to investigate the nature of the hot spots. As can be seen in the lower panel of Fig. 1, the hot spots seem to be phase locked with the density perturbation. To make this even more visible, we produced a similar spacetime diagram in which we rotated each time slice by half of the complex phase of the $m=2$ component of the density perturbation in the equatorial plane. In the following, we simply call those coordinates corotating. The result is shown in Fig. 11. The upper panel clearly demonstrates that the hot spots and the density perturbation are in phase with each other for most of the evolution. Only near the end, the picture is complicated by the decreasing amplitude of the $m=2$ mode and the growing contribution of the $m=3$ mode. Note that the sudden changes of the entropy density isocontour in the late phase of the run are not caused by strong fluid movements. On the contrary, the entropy becomes more axisymmetric and the location of the isocontour more sensitive to small fluctuations.

The stability of the hot spots in the postmerger phase raises the question why those are not dissipated by the differential rotation (cf. Fig. 9). To address this question, we investigate the fluid flow in relation to the hot spots. To get an overview, we traced fluid elements, as described in Sec. II C, and transformed their trajectories in the corotating coordinate system. The result is shown in the lower panel of Fig. 11. We find that after the merger, there are initially two large vortices with the same orientation, and a shear layer in between. For better visibility, we colored some trajectories belonging to one of the two vortices red and green, respectively. Over time, the two vortices merge into a larger one which can be interpreted as simple differential rotation on top of a large $m=2$ deformation. Note that each of the two vortices consists mainly of matter originating from one particular star, leading to the interpretation that the cores remain more or less independent for a few milliseconds.

We note that the shear layer is subject to the KelvinHelmholtz (KH) instability, which is severely underresolved at the resolutions used in our simulation. For a 

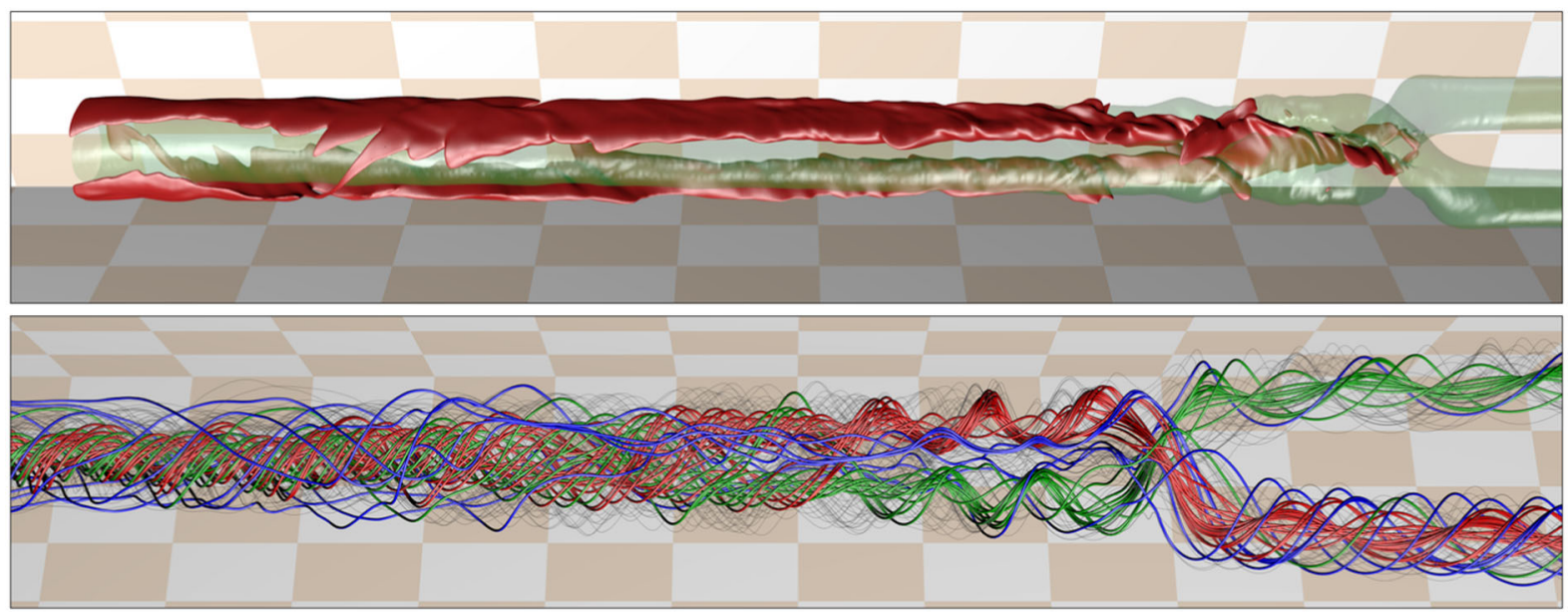

FIG. 11. Spacetime diagram showing the fluid evolution in the orbital plane. The time coordinate runs from right to left. Each time slice has been rotated to the phase of the $m=2$ component of the density perturbation in the orbital plane. The size of the tiles in the background is $2 \mathrm{~ms} \times 20 \mathrm{~km}$. Top panel: the red solid surface is the contour of constant entropy density chosen to highlight the evolution of the hot spots, and the green transparent surface is the world tube of the isodensity contour containing (at each time) $25 \%$ of the total baryon mass. Bottom panel: traced trajectories of fluid elements. Only elements not ejected from the remnant are shown. The colors highlight groups of trajectories discussed in the main text.

discussion of the KH instability in BNS mergers we refer to [53], presenting results employing the finest grid spacing to date. Here we just caution that a correct evolution of the $\mathrm{KH}$ mechanism will most likely change the time scale on which the two vortices merge, although we believe that the qualitative picture will remain unchanged.

We also found a group of fluid elements (colored blue in the figure) which move only slowly relative to the $m=2$ density perturbation. Based on Fig. 11 we find that those trajectories are roughly in the vicinity of the hot spots. It is important to know if the quasistationary trajectories agree exactly with the location of the hot spots, which would explain their stationarity with respect to the $m=2$ density perturbation. To answer this, we created another plot showing the temperature and specific entropy distribution in the equatorial plane in Fig. 12. On top of this, we plot the trajectories in the coordinates corotating with the $m=2$ perturbation, over a brief period $(4 \mathrm{~ms})$ around the time of the snapshot. We find that part of the high-temperature regions inside the remnant are indeed advected along the quasistationary fluid trajectories which form a small vortex. However, many fluid trajectories just pass through the hot spots, with the corresponding fluid elements periodically heating up and cooling down. Further, the trajectories also converge where they approach the hot spots (at least in the orbital plane). This means that part of the hot spots are dynamic in nature, generated by adiabatic temperature changes along a locally compressive flow. As further evidence, we plot the specific entropy for the same time, as shown in Fig. 13. The specific entropy in the plot varies only slightly along the trajectories crossing the hot spots. Of course, plotting trajectories on top of a snapshot showing one instant of time is only meaningful to the degree to which the thermal pattern remains stationary. By comparing to animations of the same cuts, we find that, although smaller thermal features are advected along the flow, the main hot spots are indeed largely due to the local adiabatic compression. The hot matter trapped initially in the small vortices is redistributed toward the end of the

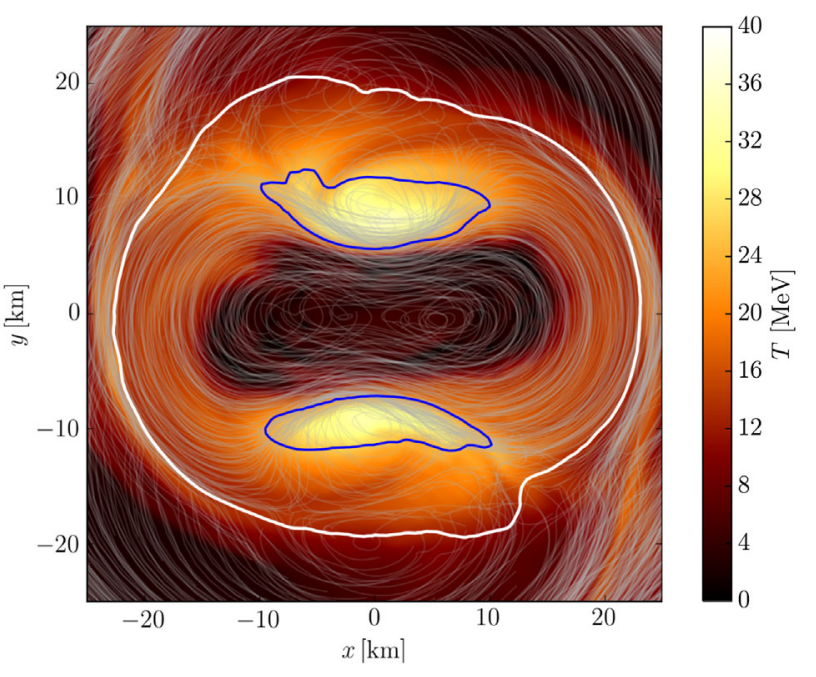

FIG. 12. Fluid motion in the frame corotating with the $m=2$ component of the density perturbation. The color plot shows the temperature in the orbital plane $8.5 \mathrm{~ms}$ after merger. The curves show fluid trajectories during the time interval $\pm 2 \mathrm{~ms}$ around the snapshot. The solid blue line marks the entropy density isocontour whose world tube is shown also in Fig. 11, and the thick white line marks the bulk isodensity surface. 


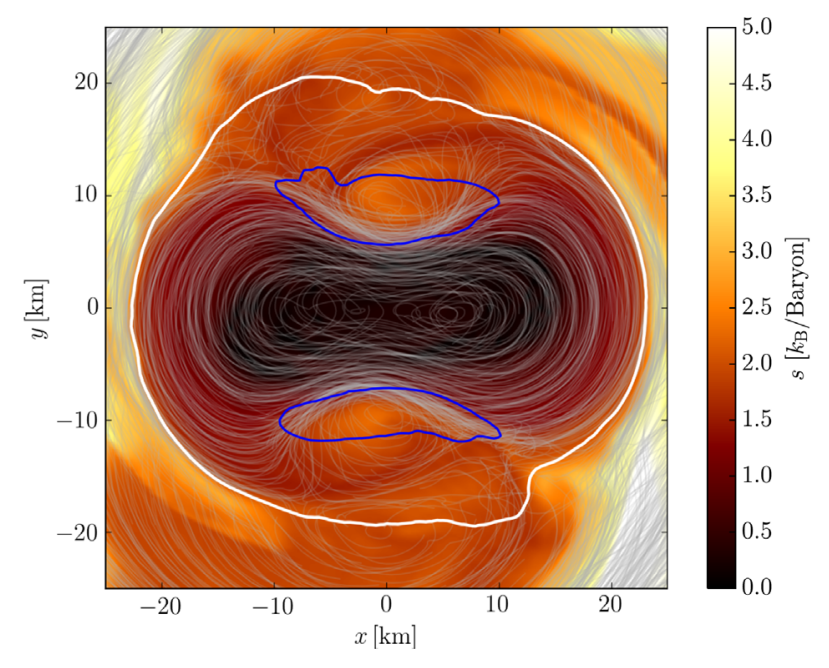

FIG. 13. Like Fig. 12, but showing the specific entropy.

simulation and becomes part of the ring-shaped structure shown in the lower right panel of Fig. 2.

\section{Gravitational waves}

In this section we present the GW signal, with focus on the postmerger signal. We are mainly interested in relating the qualitative features to the remnant dynamics. In [32], a resolution study for a different model but similar number of grid points per initial stellar radius was carried out, but could not demonstrate convergence for the complete postmerger phase. This makes it difficult to provide error estimates.

To compute the GW signal, we use an extraction radius of $916 \mathrm{~km}$ and do not extrapolate to infinity. The time integration required to obtain the strain has been carried out using the fixed-frequency integration described in [54], with a cutoff frequency of $500 \mathrm{~Hz}$. At the resolution of the coarsest grid, a wave with $3 \mathrm{kHz}$ is resolved by 10 grid points. Higher frequencies would be increasingly suppressed. However, we compared the signals extracted inside finer grids to verify that no significant high frequency signals are generated.

We find a postmerger signal dominated by the $m=2$ perturbation of the remnant. The evolution of the strain amplitude is shown in Fig. 14, in terms of the coefficient $h_{22}$ in the expansion in spin-weighted harmonics (see [55]). The strain observed at a given viewing angle $\theta$ can be obtained by multiplying with the norm of the spin weighted harmonic, $\left|{ }_{-2} Y_{22}(\theta, \phi)\right|$. We also plot the instantaneous frequency extracted from the phase of the complex strain. As one can see, the strain amplitude decays significantly within $15 \mathrm{~ms}$, as can be expected from the decrease of the $m=2$ fluid perturbation shown in Fig. 10. The frequency shows some mild oscillations after merger and then remains stable in the range $1.8-2 \mathrm{kHz}$, apart from a small drift. We note that the first peak in the instantaneous frequency is not
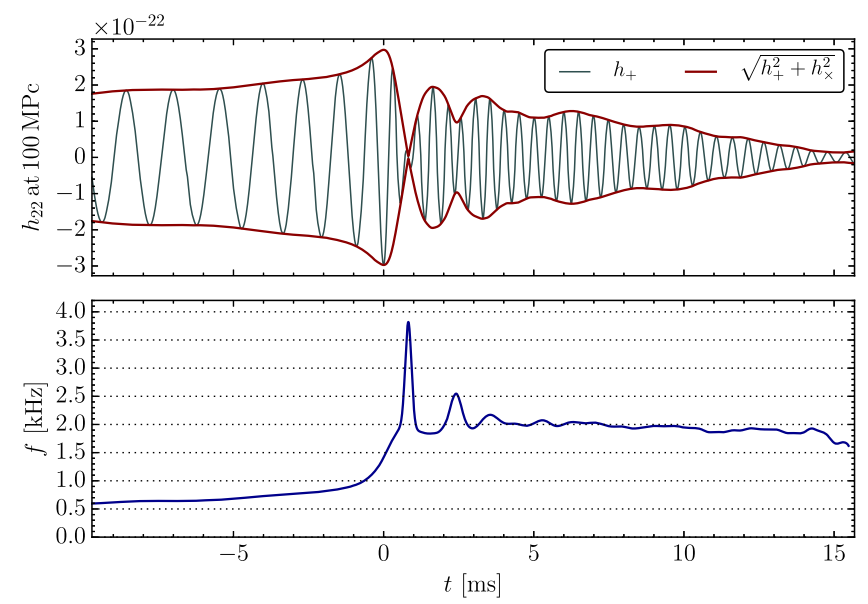

FIG. 14. Gravitational wave strain at $100 \mathrm{Mpc}$ (top panel) and instantaneous frequency (bottom panel). The latter is computed from the phase velocity of the complex strain, smoothed over $0.1 \mathrm{~ms}$ to avoid amplification of high-frequency noise.

very meaningful since the strain amplitude at this point has a rapid zero crossing.

The lower panel of Fig. 15 shows the evolution of the GW signal in a different way, plotting the strain amplitude
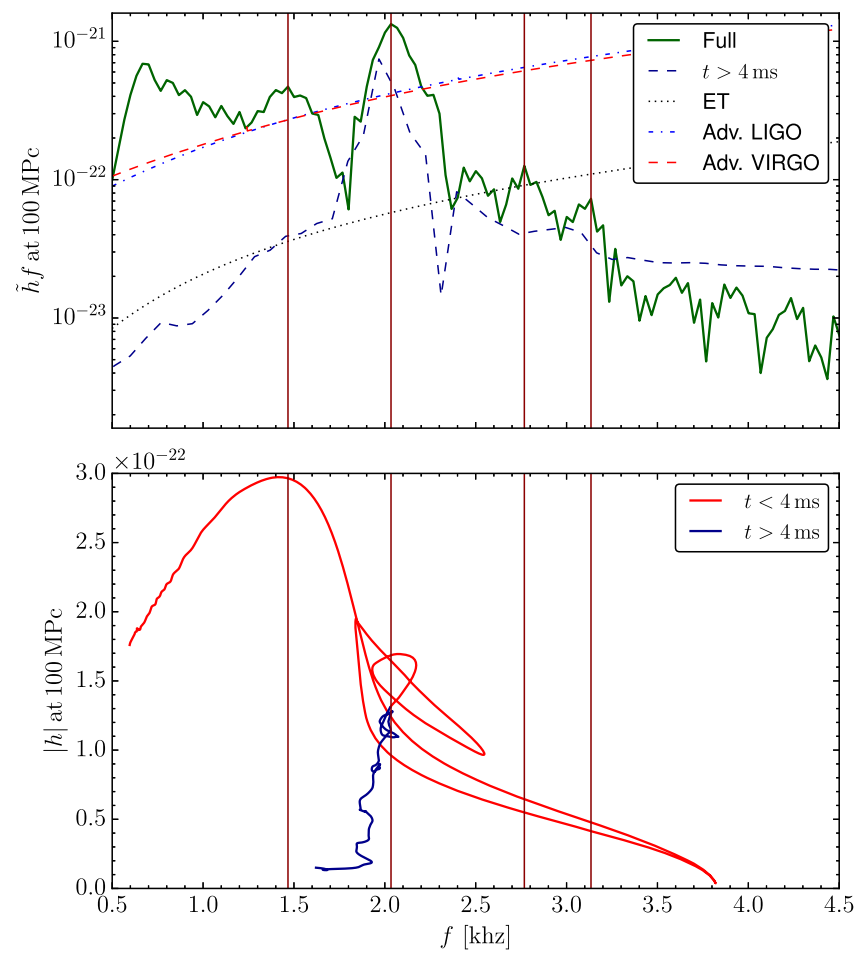

FIG. 15. Top panel: gravitational wave spectrum at $100 \mathrm{Mpc}$, both for the full simulation (solid green line) and for the strain starting $4 \mathrm{~ms}$ after the merger (dotted blue line). For comparison, we show the sensitivity curves of GW detectors. Bottom panel: strain amplitude versus instantaneous frequency. The part starting $4 \mathrm{~ms}$ after the merger is plotted in blue, the earlier evolution in red. 
versus instantaneous frequency. Both frequency and amplitude are growing during inspiral, reaching a peak amplitude at a frequency $1.4 \mathrm{kHz}$. During the merger, the frequency keeps increasing due to the increased rotation rate. At the same time, the quadrupole moment decreases as the system becomes more compact, resulting in a net decrease of strain amplitude. Next, the forming remnant reaches maximum compression, reflected in a high rotation rate. Apparently the quadrupole moment undergoes a zero crossing during this period, resulting in the brief quenching of the GW strain. Next, the remnant expands again, entering the double core phase discussed earlier. The rotation rate and thus the frequency of the GW signal decrease, while the amplitude increases because of the increasing quadrupole moment. The system then experiences a milder bounce, with an increase of frequency up to $2.5 \mathrm{kHz}$. This time the amplitude is only reduced, but stays clearly above the late-time amplitude, making this phase a significant part of the postmerger signal. Finally, the star settles down and the amplitude decreases continuously, while the frequency only shows a small drift. This late part is colored blue in the plot.

The corresponding $\mathrm{GW}$ power spectrum is shown in the top panel of Fig. 15 in comparison to the design sensitivities of current and future GW detectors. Besides the inspiral and plunge signatures, the postmerger evolution causes a prominent peak around $2 \mathrm{kHz}$. Comparing to the sensitivity curves, we find that the merger signal should be detectable by advanced LIGO/Virgo up to a distance 10-100 Mpc. We also find that if the merger itself can be detected, the postmerger signal should be visible as well. To distinguish contributions from early postmerger phase and later evolution, we computed the spectrum of the signal starting $4 \mathrm{~ms}$ after merger. We find, as expected from the instantaneous frequency, a slightly smaller peak frequency, although the difference is less than the width of the peak. More importantly, the amplitude of the peak is reduced by a factor of 2, meaning that the evolution directly after merger is an important part of the postmerger signal. We note, however, that our remnant is stable and might potentially radiate for a long time, which we did not consider in our spectra.

\section{Disk and matter ejection}

In the following, we discuss the properties of the disk surrounding the remnant. The structure in the meridional plane is shown in Fig. 16. Since the rotation rate of the remnant (compare Fig. 9) approaches the Kepler rate in the outer layers, the transition between the disk and the remnant is smooth. The plot also shows the specific entropy, which is increasing with radius, and also in the vertical direction. This agrees with Fig. 6, where we saw an increase of specific entropy with decreasing mass density. Figure 16 also shows the shape of the bulk of the remnant

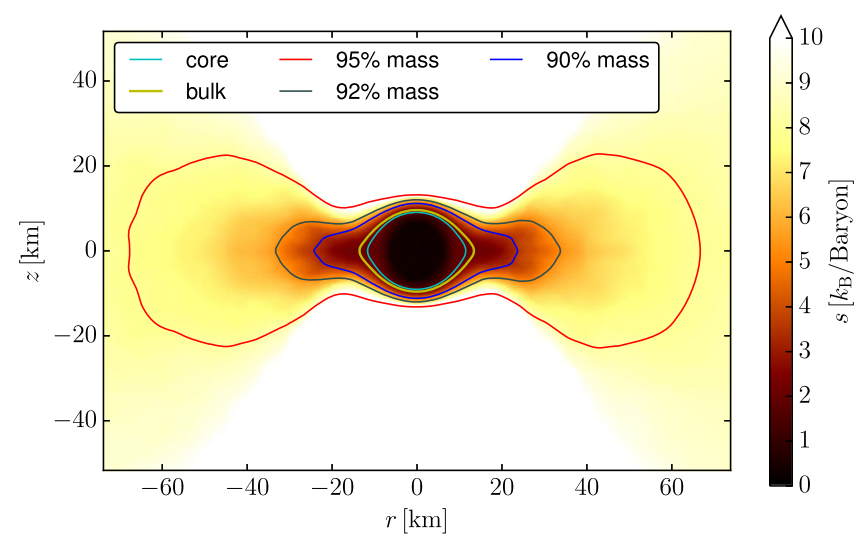

FIG. 16. Disk structure at the end of the simulation, averaged in time over $4 \mathrm{~ms}$. The colors correspond to the specific entropy, while the lines are the isocontours of mass density $1.43 \times 10^{14}$, $8.43 \times 10^{13}, 5.43 \times 10^{12}, 1.20 \times 10^{12}$, and $1.25 \times 10^{11} \mathrm{~g} / \mathrm{cm}^{3}$, from innermost to outermost, respectively. The total mass of matter with higher density than the plotted density levels is equal to the bulk mass of the TOV core equivalent, the bulk mass of the remnant itself, $90 \%, 92 \%$, and $95 \%$ of the total baryon mass, respectively.

defined in Sec. II C, demonstrating again that using the bulk properties to describe the remnant is meaningful.

Besides the structure of the disk, we also investigated its formation. To this end, we traced fluid elements backwards in time, starting from a regular grid in the orbital plane covering the disk at the end of the simulation. We then plotted the trajectories in the coordinate system corotating with the phase of the $m=2$ component of the density perturbation of the remnant. The resulting trajectories, starting $2 \mathrm{~ms}$ after merger, are shown in Fig. 17. Fluid elements that end up in the disk at the end of the simulation are colored black, while those remaining in the remnant are colored green. Since the trajectories are traced backwards in time starting with a homogeneous tracer density, the dense bundles of trajectories visible in the plot correspond to a diverging fluid flow, which is to be expected assuming that the density of a fluid element in the disk is lower compared to its point of origin inside the merging stars.

From Fig. 17, we can see that most of the matter in the disk at some point came close to the outermost parts of the strongly deformed remnant, which is not surprising. More interesting is the fact that many trajectories follow a beanshaped trajectory along the outer layers of the remnant before migrating to the disk. Also, the little vortices tailing behind the elevated parts of the deformed remnant seem to be involved in the migration of matter into the disk. It is also worth noting that apparently some of the trajectories undergo radial bounces before escaping into the disk, probably related to the radial oscillations of the remnant.

Finally, we studied the matter ejection using the methods described in Sec. IIC. The amount of ejecta is relatively small, $\approx 3 \times 10^{-4} M_{\odot}$, and is emitted mainly in a single 


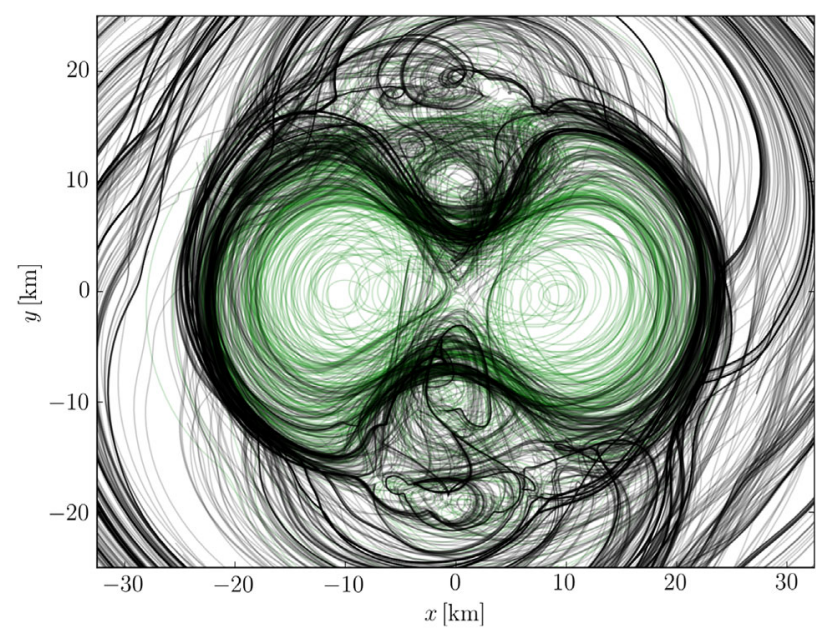

FIG. 17. Fluid trajectories in the orbital plane with respect to the coordinate system corotating with the $m=2$ density perturbation. The trajectories are shown for the time interval 2 to $12 \mathrm{~ms}$ after merger. The trajectories have been tracked backwards in time starting $14 \mathrm{~ms}$ after merger from seeds arranged in a regular grid with an extent of $70 \mathrm{~km}$. The trajectories are colored green if they stay in the remnant until the end of the simulation, or else they are black. Note the trajectories move predominantly clockwise.

wave a few milliseconds after merger. The average electron fraction is around 0.05 . We note that this reflects the electron fraction from where the ejected matter originates during the merger. Considering the neutrino radiation would most likely change the final electron fraction of the ejecta considerably (compare [56]). The average specific entropy of the ejected matter is $\approx 15 k_{B}$ per baryon, and the escape velocity $\approx 0.12 c$. We note that a binary with the same EOS but a higher total baryonic mass of $4 M_{\odot}$ was evolved in [31], and the resulting ejecta mass was around 20 times larger. This might be related to the fact that the heavier model showed stronger radial oscillations after merger.

\section{SUMMARY AND CONCLUSIONS}

In this work, we investigated the merger of two NSs, each with a mass of $1.4 M_{\odot}$, employing the ShenHorowitz-Teige EOS. Because of the atypically large maximum mass allowed by this EOS, the result is a stable NS. We followed in detail the fluid flow during and after merger and found that for around 8-10 ms, the stellar cores remain roughly independent, rotating against each other while orbiting around each other. For the first $\approx 3 \mathrm{~ms}$, they are separated by a shock-heated layer with lower density. This layer is quickly ripped apart by the fluid flow, but the cores start merging only when the shear layer in between is dissipated into smaller vortices, with an increasing number of fluid trajectories enclosing both cores. Although our resolution is not sufficient to fully resolve the KelvinHelmholtz instability, we believe the qualitative picture is correct (even if the time scales might change). After the cores have merged, the fluid flow in the equatorial plane is peanut-shaped, following a strong $m=2$ perturbation.

We studied the structure of the remnant near the end of the simulation, after it has settled down. The rotation profile has a maximum at a radius $15-20 \mathrm{~km}$, reaching a rotation rate around $960 \mathrm{~Hz}$, while the core is slowly rotating with a rate of only $240 \mathrm{~Hz}$ measured from infinity. Moreover, this rate is mostly due to frame dragging, and the core is almost nonrotating in the local inertial frame. The remnant has extended outer layers which approach Kepler velocity and smoothly join the disk orbiting the remnant.

An important open question is about the prospect for the formation of a disk surrounding the $\mathrm{BH}$ even if the collapse happens much later than the merger, so that the original disk is already accreted onto the SMNS. This has only been investigated for simplified models with $j$-const rotation law or uniform rotation [57-59]. In this respect, a rotation profile like the one described above might or might not turn out to provide the necessary conditions. Of course, this would also require that the rapid rotation of the outer bulge of the remnant persists longer than the disk. This aspect will be further investigated in future work, and in particular in relation to the "time-reversal" scenario for SGRBs [29], which envisages a late time collapse of the remnant into a $\mathrm{BH}$ plus accretion disk.

To study the mass distribution of the remnant, we introduced a new measure that replaces density profiles, mass, and compactness in a way that can be used unambiguously for rapidly and differentially rotating merger remnants without a clearly defined surface. We found that the core has a structure very similar to the core of a specific TOV star. We set up both a cold TOV solution as well as one with the same average specific entropy as the remnant, and found that, although the hot star fitted the remnant better, thermal pressure has only a little effect on the average density profile of the core.

Investigating the thermal evolution in more detail, we found that the average specific entropy of the remnant reaches $1 k_{B}$ at $5 \mathrm{~ms}$ after merger and then stays constant, while the disk is continuously heated up, reaching an average specific entropy $5 k_{B}$ at the end of the simulation. At this point, the average specific entropy at a given density raises from $0.3 k_{B}$ at the center of the remnant to around $3 k_{B}$ at the transition to the disk, and continues increasing toward lower densities. We note, however, that our simulation does not include neutrino radiation, which would likely change the temperature of the disk on the time scale of our evolution.

One of our main interests was on the inhomogeneity of the remnant temperature. We found that quickly after merger the entropy of the remnant is concentrated in two hot spots which remain stable for around $15 \mathrm{~ms}$. Those hot spots are locked in phase with the dominant $m=2$ perturbation, and consist partially of hot matter trapped in a small vortex in the "waist" of the 
peanut-shaped remnant deformation. A significant part is, however, of dynamic nature, caused by adiabatic heating along a local compression. Because of the additional thermal pressure, it seems likely that the dynamic hot spot in turn has a backreaction on the remnant deformation and hence the fluid flow. The hot spots can thus alter the amplitude of the GW signal caused by the main deformation, but, being phase locked, do not contribute additional peaks to the power spectrum for the case at hand. We note, however, that in general secondary vortices such as the ones in our model might also undergo instabilities. Our study shows that the remnant structure is clearly affected by the vortices/hot spots; hence a rearrangement might slightly alter the remnant structure and lead to a sudden change of the GW frequency.

The quantitative results we find are not general. Almost certainly results will change when considering unequal mass systems. Also the initial spin of the NSs has a strong influence, as we will show in a forthcoming publication. Nevertheless, we can conclude that the deformation of the remnant up to at least $20 \mathrm{~ms}$ after merger should be treated as a complex nonlinear quasistationary state with relevant dynamic thermal effects. A state such as the one found in our work cannot be described well in terms of linear perturbations of axisymmetric rotating stars. Since the amplitude of the postmerger GW signal is decaying, this complicated period is highly relevant for the interpretation of future detections. Interpreting minor features of the $\mathrm{GW}$ spectrum such as side peaks in terms of oscillation mode analysis developed for isolated stars might be misleading. If a postmerger GW signal is detected, its duration can be taken as a lower limit for the lifetime of the remnant. In this regard it is relevant that a mounting number of results points to slowly rotating cores, even for HMNSs and SMNSs. The lifetime is then determined by the angular momentum balance of the outer layers and disk, not the core.

\section{ACKNOWLEDGMENTS}

We acknowledge support from MIUR FIR Grant No. RBFR13QJYF. The computations in this work have been carried out on the Fermi cluster. We acknowledge the CINECA award under the ISCRA initiative, for the availability of high performance computing resources and support.
[1] G. M. Harry et al., Classical Quantum Gravity 27, 084006 (2010).

[2] F. Acernese et al., Classical Quantum Gravity 32, 024001 (2015).

[3] E. Berger, Annu. Rev. Astron. Astrophys. 52, 43 (2014).

[4] F.-K. Thielemann, A. Arcones, R. Käppeli, M. Liebendörfer, T. Rauscher, C. Winteler, C. Fröhlich, I. Dillmann, T. Fischer, G. Martinez-Pinedo, K. Langanke, K. Farouqi, K.-L. Kratz, I. Panov, and I. K. Korneev, Prog. Part. Nucl. Phys. 66, 346 (2011).

[5] S. Wanajo, Y. Sekiguchi, N. Nishimura, K. Kiuchi, K. Kyutoku, and M. Shibata, Astrophys. J. Lett. 789, L39 (2014).

[6] L.-X. Li and B. Paczyński, Astrophys. J. 507, L59 (1998).

[7] S. R. Kulkarni, arXiv:astro-ph/0510256.

[8] B. D. Metzger and E. Berger, Astrophys. J. 746, 48 (2012).

[9] N. R. Tanvir, A. J. Levan, A. S. Fruchter, J. Hjorth, R. A. Hounsell, K. Wiersema, and R. L. Tunnicliffe, Nature (London) 500, 547 (2013).

[10] E. Berger, W. Fong, and R. Chornock, Astrophys. J. Lett. 774, L23 (2013).

[11] B. Giacomazzo and R. Perna, Astrophys. J. Lett. 771, L26 (2013).

[12] D. M. Siegel and R. Ciolfi, Astrophys. J. 819, 14 (2016).

[13] D. M. Siegel and R. Ciolfi, Astrophys. J. 819, 15 (2016).

[14] Y.-Z. Fan and D. Xu, Mon. Not. R. Astron. Soc. 372, L19 (2006).
[15] Y.-W. Yu, B. Zhang, and H. Gao, Astrophys. J. Lett. 776, L40 (2013).

[16] B. D. Metzger and A. L. Piro, Mon. Not. R. Astron. Soc. 439, 3916 (2014).

[17] B. P. Abbott et al. (LIGO Scientific Collaboration and Virgo Collaboration), Phys. Rev. Lett. 116, 061102 (2016).

[18] B. P. Abbott et al. (LIGO Scientific Collaboration and Virgo Collaboration), Phys. Rev. Lett. 116, 241103 (2016).

[19] J. Abadie et al., Classical Quantum Gravity 27, 173001 (2010).

[20] A. Bauswein and H.-T. Janka, Phys. Rev. Lett. 108, 011101 (2012).

[21] K. Takami, L. Rezzolla, and L. Baiotti, Phys. Rev. Lett. 113, 091104 (2014).

[22] K. Takami, L. Rezzolla, and L. Baiotti, Phys. Rev. D 91, 064001 (2015).

[23] A. Bauswein, T. W. Baumgarte, and H.-T. Janka, Phys. Rev. Lett. 111, 131101 (2013).

[24] P. B. Demorest, T. Pennucci, S. M. Ransom, M. S. E. Roberts, and J. W. T. Hessels, Nature (London) 467, 1081 (2010).

[25] J. Antoniadis et al., Science 340, 1233232 (2013).

[26] K. Belczynski, R. O’Shaughnessy, V. Kalogera, F. Rasio, R. E. Taam, and T. Bulik, Astrophys. J. Lett. 680, L129 (2008).

[27] N. Gehrels et al., Astrophys. J. 611, 1005 (2004).

[28] A. Rowlinson, P. T. O'Brien, B. D. Metzger, N. R. Tanvir, and A. J. Levan, Mon. Not. R. Astron. Soc. 430, 1061 (2013). 
[29] R. Ciolfi and D. M. Siegel, Astrophys. J. Lett. 798, L36 (2015).

[30] T. W. Baumgarte, S. L. Shapiro, and M. Shibata, Astrophys. J. Lett. 528, L29 (2000).

[31] W. Kastaun and F. Galeazzi, Phys. Rev. D 91, 064027 (2015).

[32] A. Endrizzi, R. Ciolfi, B. Giacomazzo, W. Kastaun, and T. Kawamura, Classical Quantum Gravity 33, 164001 (2016).

[33] D. M. Siegel, R. Ciolfi, and L. Rezzolla, Astrophys. J. Lett. 785, L6 (2014).

[34] J. D. Kaplan, C. D. Ott, E. P. O’Connor, K. Kiuchi, L. Roberts, and M. Duez, Astrophys. J. 790, 19 (2014).

[35] F. Galeazzi, W. Kastaun, L. Rezzolla, and J. A. Font, Phys. Rev. D 88, 064009 (2013).

[36] N. Stergioulas, A. Bauswein, K. Zagkouris, and H.-T. Janka, Mon. Not. R. Astron. Soc. 418, 427 (2011).

[37] G. Shen, C. J. Horowitz, and S. Teige, Phys. Rev. C 82, 015806 (2010).

[38] G. Shen, C. J. Horowitz, and S. Teige, Phys. Rev. C 83, 035802 (2011).

[39] E. Gourgoulhon, P. Grandclement, K. Taniguchi, J.-A. Marck, and S. Bonazzola, Phys. Rev. D 63, 064029 (2001).

[40] T. Dietrich, N. Moldenhauer, N. K. Johnson-McDaniel, S. Bernuzzi, C. M. Markakis, B. Brügmann, and W. Tichy, Phys. Rev. D 92, 124007 (2015).

[41] D. Alic, W. Kastaun, and L. Rezzolla, Phys. Rev. D 88, 064049 (2013).

[42] D. Brown, P. Diener, O. Sarbach, E. Schnetter, and M. Tiglio, Phys. Rev. D 79, 044023 (2009).

[43] F. Löffler, J. Faber, E. Bentivegna, T. Bode, P. Diener, R. Haas, I. Hinder, B. C. Mundim, C. D. Ott, E. Schnetter, G. Allen, M. Campanelli, and P. Laguna, Classical Quantum Gravity 29, 115001 (2012).
[44] T. Nakamura, K. Oohara, and Y. Kojima, Prog. Theor. Phys. Suppl. 90, 1 (1987).

[45] M. Shibata and T. Nakamura, Phys. Rev. D 52, 5428 (1995).

[46] T. W. Baumgarte and S. L. Shapiro, Phys. Rev. D 59, 024007 (1998).

[47] D. Alic, C. Bona-Casas, C. Bona, L. Rezzolla, and C. Palenzuela, Phys. Rev. D 85, 064040 (2012).

[48] C. Bona, J. Massó, E. Seidel, and J. Stela, Phys. Rev. Lett. 75, 600 (1995).

[49] M. Alcubierre, B. Brügmann, P. Diener, M. Koppitz, D. Pollney, E. Seidel, and R. Takahashi, Phys. Rev. D 67, 084023 (2003).

[50] E. Schnetter, S. H. Hawley, and I. Hawke, Classical Quantum Gravity 21, 1465 (2004).

[51] C. Chirenti, G. H. de Souza, and W. Kastaun, Phys. Rev. D 91, 044034 (2015).

[52] S. Bernuzzi, T. Dietrich, W. Tichy, and B. Brügmann, Phys. Rev. D 89, 104021 (2014).

[53] K. Kiuchi, P. Cerdá-Durán, K. Kyutoku, Y. Sekiguchi, and M. Shibata, Phys. Rev. D 92, 124034 (2015).

[54] C. Reisswig and D. Pollney, Classical Quantum Gravity 28, 195015 (2011)

[55] K. S. Thorne, Rev. Mod. Phys. 52, 299 (1980).

[56] D. Radice, F. Galeazzi, J. Lippuner, L. F. Roberts, C. D. Ott, and L. Rezzolla, Mon. Not. R. Astron. Soc. 460, 3255 (2016).

[57] L. Baiotti, I. Hawke, and L. Rezzolla, Classical Quantum Gravity 24, S187 (2007).

[58] B. Giacomazzo, L. Rezzolla, and N. Stergioulas, Phys. Rev. D 84, 024022 (2011).

[59] B. Margalit, B. D. Metzger, and A. M. Beloborodov, Phys. Rev. Lett. 115, 171101 (2015). 